\title{
A "TYPICAL" CONTRACTION IS UNITARY
}

\author{
TANJA EISNER
}

\begin{abstract}
We show that (for the weak operator topology) the set of unitary operators on a separable infinite-dimensional Hilbert space is residual in the set of all contractions. The analogous result holds for isometries and the strong operator topology as well. These results are applied to the problem of embedding operators into strongly continuous semigroups.
\end{abstract}

\section{INTRODUCTION}

Unitary operators are the nicest and best understood operators on Hilbert spaces, and there are various results showing that there are "sufficiently many" unitary operators among the contractions. For example, the theory of Foiaş-Sz. Nagy extends every contraction to a unitary operator, called "unitary dilation", on a larger space. Furthermore, Peller [12] showed that the set of all unitary operators is dense in the set of all contractions with respect to the weak operator topology (and even for some stronger operator topology called power-weak (pw) operator topology). Finally, operator functions are bounded from above by their value on unitary operators, as proved by Nelson [11].

In this note we improve the above density result of Peller and show that on a (separable infinite-dimensional) Hilbert space the unitary operators form a residual subset of the set of all contractions (isometries) with respect to the weak (strong) operator topology, see Section 1 and 2. (Recall that a set of a Baire space is called residual if its complement is of first category.) In this sense, a "typical" contraction or isometry is unitary.

We apply this result to the problem of embedding an operator into a strongly continuous semigroup, see Section 3. We show that a "typical" contraction or isometry on a separable infinite-dimensional Hilbert space is embeddable. This is an operator-theoretic counterpart to a recent result from ergodic theory on embedding a measure-preserving transformation into a flow, see de la Rue, de Sam Lazaro [13]. In particular, a "typical" contraction has roots of all order, extending the analogous result from ergodic theory, see King [9].

\section{ISOMETRIES}

Let $H$ be an infinite-dimensional separable Hilbert space. We denote by $\mathcal{U}$ the set of all unitary operators and by $\mathcal{I}$ the set of all isometries on $H$ endowed with the strong operator topology. We show in this section that $\mathcal{U}$ is residual in $\mathcal{I}$, i.e., its complement $\mathcal{I} \backslash \mathcal{U}$ is of first category. This shows that a "typical" isometry is unitary.

The space $\mathcal{I}$ is a complete metric space with respect to the metric

$$
d(T, S):=\sum_{j=1}^{\infty} \frac{\left\|T x_{j}-S x_{j}\right\|}{2^{j}\left\|x_{j}\right\|} \quad \text { for } T, S \in \mathcal{I},
$$

2000 Mathematics Subject Classification. 47A05, 47D06, 37A05.

Key words and phrases. Unitary operators, contractions, Baire category, $C_{0}$-semigroups. 
where $\left\{x_{j}\right\}_{j=1}^{\infty}$ is a fixed dense subset of $H \backslash\{0\}$.

The starting point is the following lemma based on the Wold decomposition of an isometry, see e.g. Eisner, Sereny [3].

Lemma 2.1. The set $\mathcal{U}$ of unitary operators is dense in $\mathcal{I}$.

However, the following much stronger result holds.

Theorem 2.2. The set $\mathcal{U}$ of unitary operators is residual in $\mathcal{I}$.

Proof. Let $T$ be a non-invertible isometry. Then $\operatorname{rg} T$ is closed and different from $H$. Therefore, there exists $x_{j}$ with $\operatorname{dist}\left(x_{j}, \operatorname{rg} T\right)>0$, hence

$$
\mathcal{I} \backslash \mathcal{U}=\bigcup_{k, j=1}^{\infty} M_{j, k} \quad \text { with } \quad M_{i, k}:=\left\{T: \operatorname{dist}\left(x_{j}, \operatorname{rg} T\right)>\frac{1}{k}\right\} .
$$

We prove now that every set $M_{j, k}$ is nowhere dense in $\mathcal{I}$. By Lemma 2.1 it suffices to show that

$$
\mathcal{U} \cap \overline{M_{j, k}}=\emptyset \quad \forall j, k
$$

Assume the contrary, i.e., that there exists a sequence $\left\{T_{n}\right\}_{n=1}^{\infty} \subset M_{j, k}$ for some $j, k$ and a unitary operator $U$ with $\lim _{n \rightarrow \infty} T_{n}=U$ strongly. In particular, $\lim _{n \rightarrow \infty} T_{n} y=U y=x_{j}$ for $y:=U^{-1} x_{j}$. This however implies $\lim _{n \rightarrow \infty} \operatorname{dist}\left(x_{j}, \operatorname{rg} T_{n}\right)=0$, a contradiction. So (1) is proved, every set $M_{j, k}$ is nowhere dense, and $\mathcal{U}$ is residual in $\mathcal{I}$.

\section{Contractions}

As before, we consider a separable infinite-dimensionals Hilbert space $H$ and prove now that a "typical" contraction on $H$ is unitary.

Denote by $\mathcal{C}$ the set of all contractions on $H$ endowed with the weak operator topology. This is a complete metric space with respect to the metric

$$
d(T, S):=\sum_{i, j=1}^{\infty} \frac{\left|\left\langle T x_{i}, x_{j}\right\rangle-\left\langle S x_{i}, x_{j}\right\rangle\right|}{2^{i+j}\left\|x_{i}\right\|\left\|x_{j}\right\|} \text { for } T, S \in \mathcal{C},
$$

where $\left\{x_{j}\right\}_{j=1}^{\infty}$ is a fixed dense subset of $H \backslash\{0\}$.

The following density result holds for unitary operators, see Peller [12] (for a much stronger result) or Eisner, Serény [3].

Lemma 3.1. The set $\mathcal{U}$ of unitary operators is dense in $\mathcal{C}$.

Our construction uses the following well-known property of weak convergence, see e.g. Halmos [8]. For the reader's convenience we give its simple proof.

Lemma 3.2. Let $\left\{T_{n}\right\}_{n=1}^{\infty}$ be a sequence of linear operators on a Hilbert space $H$ converging weakly to a linear operator $S$. If $\left\|T_{n} x\right\| \leq\|S x\|$ for every $x \in H$, then $\lim _{n \rightarrow \infty} T_{n}=S$ strongly.

Proof. For every $x \in H$ we have

$$
\begin{aligned}
\left\|T_{n} x-S x\right\|^{2} & =\left\langle T_{n} x-S x, T_{n} x-S x\right\rangle=\|S x\|^{2}+\left\|T_{n} x\right\|^{2}-2 \operatorname{Re}\left\langle T_{n} x, S x\right\rangle \\
& \leq 2\langle S x, S x\rangle-2 \operatorname{Re}\left\langle T_{n} x, S x\right\rangle=2 \operatorname{Re}\left\langle\left(S-T_{n}\right) x, S x\right\rangle \rightarrow 0 \text { as } n \rightarrow \infty,
\end{aligned}
$$

and the lemma is proved. 
We now state the main result of this paper.

Theorem 3.3. The set $\mathcal{U}$ of unitary operators is residual in the set $\mathcal{C}$ of contractions.

Proof. We first prove that the set $\mathcal{I} \backslash \mathcal{U}$ of non-invertible isometries is of first category. As in the proof of Theorem $2.2, \mathcal{I} \backslash \mathcal{U}$ is given as

$$
\mathcal{I} \backslash \mathcal{U}=\bigcup_{k, j=1}^{\infty} M_{j, k} \quad \text { with } \quad M_{i, k}:=\left\{T \text { isometric }: \operatorname{dist}\left(x_{j}, \operatorname{rg} T\right)>\frac{1}{k}\right\} .
$$

By Lemma 3.1 it is enough to show that

$$
\mathcal{U} \cap \overline{M_{j, k}}=\emptyset \quad \forall j, k .
$$

Assume that for some $j, k$ there exists a sequence $\left\{T_{n}\right\}_{n=1}^{\infty} \subset M_{j, k}$ converging weakly to a unitary operator $U$. Then, by Lemma 3.2, $T_{n}$ converges to $U$ strongly. As in the proof of Theorem 2.2 , this implies $\lim _{n \rightarrow \infty} T_{n} y=U y=x_{j}$ for $y:=U^{-1} x_{j}$. Hence $\lim _{n \rightarrow \infty} \operatorname{dist}\left(x_{j}, \operatorname{rg} T_{n}\right)=0$ contradicting $\left\{T_{n}\right\}_{n=1}^{\infty} \subset M_{j, k}$, so every $M_{j, k}$ is nowhere dense and $\mathcal{I} \backslash \mathcal{U}$ is of first category.

We now show that the set of non-isometric operators is of first category in $\mathcal{C}$ as well. Let $T$ be a non-isometric contraction. Then there exists $x_{j}$ such that $\left\|T x_{j}\right\|<\left\|x_{j}\right\|$, hence

$$
\mathcal{C} \backslash \mathcal{I}=\bigcup_{k, j=1}^{\infty} N_{j, k} \quad \text { with } \quad N_{j, k}:=\left\{T: \frac{\left\|T x_{j}\right\|}{\left\|x_{j}\right\|}<1-\frac{1}{k}\right\} .
$$

It remains to show that every $N_{j, k}$ is nowhere dense in $\mathcal{C}$. By Lemma 3.1 again it suffices to show

$$
\mathcal{U} \cap \overline{N_{j, k}}=\emptyset \quad \forall j, k .
$$

Assume that for some $j, k$ there exists a sequence $\left\{T_{n}\right\}_{n=1}^{\infty} \subset N_{j, k}$ converging weakly to a unitary operator $U$. Then $T_{n}$ converges to $U$ strongly by Lemma 3.2. This implies in particular that $\lim _{n \rightarrow \infty}\left\|T_{n} x_{j}\right\|=\left\|U x_{j}\right\|=\left\|x_{j}\right\|$ contradicting $\frac{\left\|T_{n} x_{j}\right\|}{\left\|x_{j}\right\|}<1-\frac{1}{k}$ for every $n \in \mathbb{N}$.

Remark 3.4. Peller [12] showed that the set of unitary operators is dense in the set of contractions with respect to the so-called pw-topology (power-weak operator topology). This topology corresponds to weak convergence of all powers, i.e.,

$$
\text { pw-lim } T_{n}=S \quad \Longleftrightarrow \quad \lim _{n \rightarrow \infty} T_{n}^{k}=S^{k} \text { weakly for all } k \in \mathbb{N} \text {. }
$$

It is natural to ask whether unitary operators are residual with respect to this topology as well. Indeed, all the arguments used in the proof of Theorem 3.3 remain valid for this topology, hence the complement of $\mathcal{U}$ is of first category. However, the space of all contractions is no longer complete, see Eisner, Serény [4] for a diverging Cauchy sequence. So it is not clear whether $\mathcal{C}$ with the pw-topology is a Baire space.

\section{Applichtion to the embedding problem}

In this section we consider the following problem: Which bounded operators $T$ can be embedded into a strongly continuous semigroup, i.e., does there exist a $C_{0}$-semigroup $(T(t))_{t \geq 0}$ with $T=T(1)$ ? (For the basic theory of $C_{0}$-semigroups we refer to Engel, Nagel [5].) For some classes of operators this question has a positive answer, e.g., for operators with spectrum in a certain area using functional calculus, see e.g. Haase [6, Section 3.1], and for isometries on Hilbert spaces with infinite-dimensional kernel, see [2]. 
We apply the above category results to this problem and show that a "typical" isometry and a "typical" contraction on a separable infinite-dimensional Hilbert space is embeddable.

It is well-known that unitary operators have the embedding property.

Lemma 4.1. Every unitary operator on a Hilbert space can be embedded into a unitary $C_{0}$-group.

Proof. Let $T$ be a unitary operator on a Hilbert space $H$. Then by the spectral theorem, see e.g. Halmos [7], $T$ is isomorphic to a direct sum of multiplication operators $M$ given by $M f\left(e^{i \varphi}\right):=e^{i \varphi} f\left(e^{i \varphi}\right)$ on $L^{2}(\Gamma, \mu)$ for the unit circle $\Gamma$ and some measure $\mu$. Each such operator can be embedded into the unitary $C_{0}$-group $(U(t))_{t \in \mathbb{R}}$ given by

$$
U(t) f\left(e^{i \varphi}\right):=e^{i t \varphi} f\left(e^{i \varphi}\right), \quad \varphi \in[0,2 \pi], t \in \mathbb{R} .
$$

A direct corollary of Theorem 2.2, Theorem 3.3 and the above lemma is the following category result for embeddable operators.

Theorem 4.2. On a separable infinite-dimensional Hilbert space, the set of all embeddable operators is residual in the set $\mathcal{I}$ of all isometries (for the strong operator topology) and in the set $\mathcal{C}$ of all contractions (for the weak operator topology).

In other words, a "typical" isometry and a "typical" contraction can be embedded into a $C_{0}$-semigroup. This is an operator theoretic counterpart to a recent result of de la Rue, de Sam Lazaro [13] in ergodic theory stating that a "typical" measure preserving transformation can be embedded into a continuous measure preserving flow.

Remark 4.3. In particular, a "typical" contraction (on a separable infinite-dimensional Hilbert space) has roots of every order. This is an operator theoretic analogue of a result of King [9] from ergodic theory. See also Ageev [1] and Stepin, Eremenko [14] for related results.

Acknowledgement. The author is grateful to Rainer Nagel for valuable comments.

\section{REFERENCES}

[1] O. N. Ageev, On the genericity of some non-asymptotic dynamical properties, Uspekhi Mat. Nauk 58 (2003), 177-178; translation in Russian Math. Surveys 58 (2003), 173-174.

[2] T. Eisner, Embedding linear operators into strongly continuous semigroups, preprint.

[3] T. Eisner and A. Serény, Category theorems for stable operators on Hilbert spaces, Acta Sci. Math. (Szeged) 74 (2008), 259-270.

[4] T. Eisner and A. Serény, On the weak analogue of the Trotter-Kato theorem, submitted.

[5] K.-J. Engel and R. Nagel, One-parameter Semigroups for Linear Evolution Equations, Graduate Texts in Mathematics, vol. 194, Springer-Verlag, New York, 2000.

[6] M. Haase, The Functional Calculus for Sectorial Operators, Operator Theory: Advances and Applications, vol. 169, Birkhäuser Verlag, Basel, 2006.

[7] P. R. Halmos, What does the spectral theorem say?, Amer. Math. Monthly 70 (1963), 241-247.

[8] P. R. Halmos, A Hilbert Space Problem Book, D. Van Nostrand Co., Inc., Princeton, N.J.-Toronto, Ont.London 1967.

[9] J. L. King, The generic transformation has roots of all orders, Colloq. Math. 84/85 (2000), 521-547.

[10] R. Nagel (ed.), One-parameter Semigroups of Positive Operators, Lecture Notes in Mathematics, vol. 1184, Springer-Verlag, Berlin, 1986.

[11] E. Nelson, The distinguished boundary of the unit operator ball, Proc. Amer. Math. Soc. 12 (1961), 994-995. 
[12] V. V. Peller, Estimates of operator polynomials in the space $L^{p}$ with respect to the multiplicative norm, J. Math. Sciences 16 (1981), 1139-1149.

[13] T. de la Rue and J. de Sam Lazaro, The generic transformation can be embedded in a flow (French), Ann. Inst. H. Poincaré, Prob. Statist. 39 (2003), 121-134.

[14] A. M. Stepin, A. M. Eremenko, Nonuniqueness of an inclusion in a flow and the vastness of a centralizer for a generic measure-preserving transformation, Mat. Sb. 195 (2004), 95-108; translation in Sb. Math. 195 (2004), 1795-1808.

[15] B. Sz.-Nagy and C. Foiaş, Harmonic Analysis of Operators on Hilbert Space, North-Holland Publishing Co., 1970.

Mathematisches Institut, Universität Tübingen

Auf Der Morgenstelle 10, D-70176, Tübingen, Germany

E-mail address: talo@fa.uni-tuebingen.de 